\title{
A combinatorial formula for Earle's twisted 1-cocycle on the mapping class group $\mathcal{M}_{g, *}$
}

\author{
Yusuke Kuno
}

\begin{abstract}
We present a formula expressing Earle's twisted 1-cocycle on the mapping class group of a closed oriented surface of genus $\geq 2$ relative to a fixed base point, with coefficients in the first homology group of the surface. For this purpose we compare it with Morita's twisted 1-cocycle which is combinatorial. The key is the computation of these cocycles on a particular element of the mapping class group, which is topologically a hyperelliptic involution.
\end{abstract}

Introduction and statement of the result. Let $\left(\Sigma_{g}, *\right)$ be a closed oriented $C^{\infty}$ surface of genus $\geq 2$ with a fixed base point $*$ and let $\mathcal{M}_{g, *}$ be the mapping class group of $\left(\Sigma_{g}, *\right)$, namely the group of all orientation preserving diffeomorphisms of $\left(\Sigma_{g}, *\right)$ modulo isotopies fixing the base point $*$. The group $\mathcal{M}_{g, *}$ naturally acts on the first homology group $H=H_{1}\left(\Sigma_{g} ; \mathbb{Z}\right)$.

In [1], C. Earle discovered a twisted 1-cocycle $\psi: \mathcal{M}_{g, *} \rightarrow \frac{1}{2 g-2} H$. This cocycle is complex analytic by nature. In fact, he discovered this cocycle in the study of the action of $\mathcal{M}_{g, *}=\bmod (\Gamma)$ on $J(V)$, using his notation, the family of Jacobi varieties over the Teichmüller space of compact Riemann surfaces of genus $g$. We call $\psi$ Earle's twisted 1-cocycle. The construction of $\psi$ will be recalled in section 2 .

In view of [3], $\psi$ gives rise to a generator of the first cohomology group $H^{1}\left(\mathcal{M}_{g, *} ; H\right) \cong$ $H^{1}\left(\mathcal{M}_{g, 1} ; H\right) \cong \mathbb{Z}$. Here $\mathcal{M}_{g, 1}$ is the mapping class group of $\Sigma_{g}$ relative to an embedded disc, see section 1. Amusingly, other than $\psi$ there have been known various ways of constructing cocycles representing a generator of this cohomology group; see S. Morita [3, 4, 5, 6] and T. Trapp [7]. Among others, there is a combinatorial one: Morita's twisted 1-cocycle $f: \mathcal{M}_{g, *} \rightarrow H$ defined in [3].

Although $\psi$ naturally arises it seems more abstract than other known cocycles. At first glance, the construction of $\psi$ does not tell much about its value on a given element of $\mathcal{M}_{g, *}$ which is, for example, expressed as a product of Dehn twists. The aim of the present paper is to improve this unsatisfactory situation. For this purpose we compare $\psi$ with $f$. As a product we obtain a formula expressing Earle's cocycle $\psi$, which appeared in the context of complex analysis, using Morita's cocycle $f$, more combinatorial one.

To state the result, let us fix the notation. Let $A_{1}, \ldots, A_{g}, B_{1}, \ldots, B_{g}$ be a fundamental system of generators of the fundamental group $\pi_{1}\left(\Sigma_{g}, *\right)$ and fix it throughout this paper. Then the group $\pi_{1}\left(\Sigma_{g}, *\right)$ is isomorphic to the group

$$
\Gamma=<A_{1}, \ldots, A_{g}, B_{1}, \ldots, B_{g} \mid \zeta=1>.
$$


Here, $\zeta=\prod_{k=1}^{g}\left[A_{k}, B_{k}\right]=\left[A_{1}, B_{1}\right] \cdots\left[A_{g}, B_{g}\right]$ and $\left[A_{k}, B_{k}\right]=A_{k} B_{k} A_{k}^{-1} B_{k}^{-1}$. The natural projection $\theta: \Gamma \rightarrow H$ gives the abelianization of $\Gamma$ and $H$ is identified with $\mathbb{Z}^{2 g}$ by the direct decomposition

$$
H=\mathbb{Z} \cdot \theta\left(A_{1}\right) \oplus \cdots \oplus \mathbb{Z} \cdot \theta\left(B_{g}\right) .
$$

We denote the action of $\mathcal{M}_{g, *}$ on $H$ by $\rho: \mathcal{M}_{g, *} \rightarrow S p(H)$. Here $S p(H)$ denotes the group of automorphisms of $H$ preserving the intersection form. In view of the identification $H \cong \mathbb{Z}^{2 g}$ given above, $S p(H)$ is identified with the symplectic group $S p(2 g ; \mathbb{Z})$. Let $a_{0} \in$ $\frac{1}{2 g-2} \mathbb{Z}^{2 g}$ be the column vector defined by

$$
a_{0}=\frac{1}{2 g-2}{ }^{t}(\underbrace{0, \ldots, 0}_{g}, \underbrace{2, \ldots, 2}_{g}) .
$$

The result of this paper is:

Theorem 0.1. We have

$$
\psi=-\frac{1}{2 g-2} f+\delta a_{0},
$$

where the coboundary $\delta a_{0}$ is given by $\delta a_{0}(\phi)=\rho(\phi)^{-1} \cdot a_{0}-a_{0}, \phi \in \mathcal{M}_{g, *}$.

The organization of this paper is as follows. In section 1 we introduce some groups other than $\mathcal{M}_{g, *}$ and recall a result on $H^{1}\left(\mathcal{M}_{g, *} ; H\right)$ by Morita. Sections 2 and 3 are devoted to the review of Earle's cocycle and Morita's cocycle, respectively. In section 4 a particular element $\bar{\iota} \in \mathcal{M}_{g, *}$, which is topologically a hyperelliptic involution, is defined. Theorem 0.1 will be proved in section 5 . The key lemma to prove Theorem 0.1 is Lemma 5.2 in which we compute the value $\psi(\bar{\iota})$ explicitly. This computation will be performed in section 6 , in which we also prove Lemma 5.1, another lemma needed to the proof of Theorem 0.1 .

\section{Mapping class groups, $H^{1}\left(\mathcal{M}_{g, *} ; H\right)$}

We first introduce some groups related to $\mathcal{M}_{g, *}$. Let $D \subset \Sigma_{g}$ be an embedded closed 2-disk centered at $*$ and let $\mathcal{M}_{g, 1}$ be the mapping class group of $\left(\Sigma_{g}, D\right)$, namely the group of all orientation preserving diffeomorphisms of $\left(\Sigma_{g}, D\right)$ modulo isotopies fixing $D$. Choose a base point $*^{\prime}$ of $\Sigma_{g} \backslash \operatorname{Int} D$ in $\partial D$. The fundamental group $\pi_{1}\left(\Sigma_{g} \backslash \operatorname{Int} D, *^{\prime}\right)$ is isomorphic to a free group of rank $2 g$. By an appropriate choice of (homotopy class of) based loops $A_{1}^{\prime}, \ldots, A_{g}^{\prime}, B_{1}^{\prime}, \ldots, B_{g}^{\prime}$ in $\Sigma_{g} \backslash \operatorname{Int} D$, we can arrange that;

1. $\pi_{1}\left(\Sigma_{g} \backslash \operatorname{Int} D, *^{\prime}\right)$ is freely generated by $A_{1}^{\prime}, \ldots, A_{g}^{\prime}, B_{1}^{\prime}, \ldots, B_{g}^{\prime}$.

2. $\zeta^{\prime}=\prod_{k=1}^{g}\left[A_{k}^{\prime}, B_{k}^{\prime}\right]$ is homotopic rel $*^{\prime}$ to the boundary loop $\partial D$.

3. Let $\gamma$ be a path in $D$ joining $*$ and $*^{\prime}$. Joining with $\gamma$, we have a natural homomorphism from $\pi_{1}\left(\Sigma_{g} \backslash \operatorname{Int} D, *^{\prime}\right)$ to $\pi_{1}\left(\Sigma_{g}, *\right)$. Then for each $k, A_{k}^{\prime}$ (resp. $\left.B_{k}^{\prime}\right)$ is mapped to $A_{k}$ (resp. $\left.B_{k}\right)$.

Henceforth we write $A_{k}$ (resp. $B_{k}$ ) instead of $A_{k}^{\prime}$ (resp. $B_{k}^{\prime}$ ) for simplicity. Let $F=$ $F\left(A_{1}, \ldots, A_{g}, B_{1}, \ldots, B_{g}\right)$ be the free group generated by $A_{1}, \ldots, A_{g}, B_{1}, \ldots, B_{g}$. The mapping class groups $\mathcal{M}_{g, 1}$ and $\mathcal{M}_{g, *}$ act on the fundamental groups of the surfaces. By the 
well-known theorem of Dehn-Nielsen, these actions are faithful and we have the isomorphisms

$$
\mathcal{M}_{g, 1} \stackrel{\cong}{\rightarrow}\{\phi \in \operatorname{Aut}(F) ; \phi(\zeta)=\zeta\}
$$

and

$$
\mathcal{M}_{g, *} \stackrel{\cong}{\rightarrow} \operatorname{Aut}^{+}(\Gamma)
$$

where ${ }^{+}$means acting on the second homology $H_{2}(\Gamma) \cong \mathbb{Z}$ as the identity. We identify $\mathcal{M}_{g, 1}$ (resp. $\mathcal{M}_{g, *}$ ) with its image of the above isomorphism. Note that these identifications depend on the choice of a fundamental system of generators of $\pi_{1}\left(\Sigma_{g}, *\right)$. We also consider the subgroup $\mathcal{N}$ of $A u t(F)$, including $\mathcal{M}_{g, 1}$, defined by

$$
\mathcal{N}:=\{\phi \in A u t(F) ; \phi(\zeta) \text { is conjugate to } \zeta\} .
$$

Each element of $\mathcal{N}$ induces an automorphism of $\Gamma$ acting on $H_{2}(\Gamma)$ as the identity. Thus we have a homomorphism

$$
\pi: \mathcal{N} \rightarrow \operatorname{Aut}^{+}(\Gamma) \cong \mathcal{M}_{g, *},
$$

and it is well known that the restriction of $\pi$ to $\mathcal{M}_{g, 1}$ gives rise to the central extension

$$
0 \rightarrow \mathbb{Z} \rightarrow \mathcal{M}_{g, 1} \stackrel{\left.\pi\right|_{\mathcal{M}_{g, 1}}}{\rightarrow} \mathcal{M}_{g, *} \rightarrow 1
$$

Topologically, $\left.\pi\right|_{\mathcal{M}_{g, 1}}$ is induced by regarding diffeomorphisms of $\left(\Sigma_{g}, D\right)$ as diffeomorphisms of $\left(\Sigma_{g}, *\right)$, and the generator of the kernel corresponds to the Dehn twist along the boundary $\zeta=\partial D$.

We next recall a result on $H^{1}\left(\mathcal{M}_{g, *} ; H\right)$. First, let us introduce the conventions in this paper. Let $G$ be a group and $M$ a (left) $G$-module. By a twisted 1-cocycle is meant a map $\Phi: G \rightarrow M$ satisfying

$$
\Phi\left(\gamma_{1} \gamma_{2}\right)=\gamma_{2}^{-1} \cdot \Phi\left(\gamma_{1}\right)+\Phi\left(\gamma_{2}\right)
$$

for all $\gamma_{1}, \gamma_{2} \in G$. For $m \in M$, by the coboundary of $m$ is meant the map $\delta m: G \rightarrow M$ defined by

$$
\delta m(\gamma)=\gamma^{-1} \cdot m-m
$$

As usual coboundaries are twisted 1-cocycles. The quotient

$$
\text { \{twisted } 1 \text {-cocycles }\} /\{\text { coboundaries }\}
$$

is denoted by $H^{1}(G ; M)$ and called the first cohomology group of $G$ with coefficients in $M$.

Regard the group $\pi_{1}\left(\Sigma_{g}, *\right)$ as a subgroup of $\mathcal{M}_{g, *}$ via inner automorphism; For $x \in$ $\pi_{1}(\Sigma, *) \cong \Gamma$, the map $\Gamma \rightarrow \Gamma, y \mapsto x y x^{-1}$ is the corresponding element of $\mathcal{M}_{g, *} \cong A_{u t}+(\Gamma)$. In [3], Morita determined the cohomology group $H^{1}\left(\mathcal{M}_{g, *} ; H\right)$ :

Proposition 1.1 (Morita [3]). The cohomology group $H^{1}\left(\mathcal{M}_{g, *} ; H\right)$ is isomorphic to the infinite cyclic group $\mathbb{Z}$. A twisted 1-cocycle $\Phi: \mathcal{M}_{g, *} \rightarrow H$ represents a generator of $H^{1}\left(\mathcal{M}_{g, *} ; H\right)$ if and only if the restriction of $\Phi$ to $\pi_{1}\left(\Sigma_{g}, *\right)$ coincides with $\pm(2 g-2)$ times the abelianization: $\left.\Phi\right|_{\pi_{1}\left(\Sigma_{g}, *\right)}= \pm(2 g-2) \theta$. 


\section{Earle's twisted 1-cocycle}

We review Earle's twisted 1-cocycle $\psi$ described in [1]. Let $\mathcal{T}_{g, 1}$ be the Teichmüller space of compact Riemann surfaces of genus $g$ with one distinguished point; It is the set of equivalence classes of all triads $(X, p, f)$ such that

1. $X$ is a compact Riemann surface of genus $g$,

2. $f:\left(\Sigma_{g}, *\right) \rightarrow(X, p)$ is an orientation preserving diffeomorphism,

and the equivalence relation is defined as follows: $(X, p, f)$ and $\left(X^{\prime}, p^{\prime}, f^{\prime}\right)$ are equivalent if there exists a biholomorphic map $h:(X, p) \rightarrow\left(X^{\prime}, p^{\prime}\right)$ such that $h \circ f$ is homotopic to $f^{\prime}$. We denote by $[X, p, f]$ the point of $\mathcal{T}_{g, 1}$ represented by $(X, p, f)$. The mapping class group $\mathcal{M}_{g, *}$ acts on $\mathcal{T}_{g, 1}$ by

$$
\phi \cdot[X, p, f]=\left[X, p, f \circ \phi^{-1}\right],
$$

where $\phi \in \mathcal{M}_{g, *}$ and $[X, p, f] \in \mathcal{T}_{g, 1}$. $\mathcal{T}_{g, 1}$ has the natural complex structure and is isomorphic to the Bers fiber space.

Now the holomorphic map $\eta: \mathcal{T}_{g, 1} \rightarrow \mathbb{C}^{g}$ is defined as follows. Let $[X, p, f]$ be an element of $\mathcal{T}_{g, 1}$. Then $f_{*} A_{1}, \ldots, f_{*} A_{g}, f_{*} B_{1}, \ldots, f_{*} B_{g}$ is a fundamental system of generators of $\pi_{1}(X, p)$. Let $\omega_{1}, \ldots, \omega_{g}$ be the basis of holomorphic 1-forms on $X$ satisfying the normalized condition

$$
\int_{\left[f_{*} A_{i}\right]} \omega_{j}=\delta_{i j}
$$

where $\delta_{i j}$ is the Kronecker delta and $[T] \in H_{1}(X ; \mathbb{Z})$ denotes the homology class represented by the loop $T$. Set $\tau_{i j}=\int_{\left[f_{*} B_{i}\right]} \omega_{j}$. The $g \times g$ matrix $\tau=\left(\tau_{i j}\right)_{i, j}$ is the period matrix of $X$ with respect to the symplectic basis $\left[f_{*} A_{1}\right], \ldots,\left[f_{*} A_{g}\right],\left[f_{*} B_{1}\right], \ldots,\left[f_{*} B_{g}\right]$.

For each pair $(j, k)(1 \leq j, k \leq g)$, the quadratic period class corresponding to $\omega_{j}$ and $\omega_{k}$ is the function $Q_{j k}: \pi_{1}(X, p) \rightarrow \mathbb{C}$ defined by

$$
Q_{j k}(T):=\int_{T} \omega_{k} \omega_{j}=\int_{s=0}^{1} \omega_{k}(T(s)) \int_{u=0}^{s} \omega_{j}(T(u)),
$$

where $T \in \pi_{1}(X, p)$. Finally, define $\eta={ }^{t}\left(\eta_{1}, \ldots, \eta_{g}\right)$ by

$$
(1-g) \eta_{j}([X, p, f]):=-\frac{1}{2} \tau_{j j}+\sum_{k=1}^{g} Q_{j k}\left(f_{*} A_{k}\right), 1 \leq j \leq g .
$$

It is known that $(1-g) \eta([X, p, f])$ is the vector of Riemann constants for $(X, p)$ with respect to the symplectic basis $\left[f_{*} A_{1}\right], \ldots,\left[f_{*} A_{g}\right],\left[f_{*} B_{1}\right], \ldots,\left[f_{*} B_{g}\right]$.

For $\phi \in \mathcal{M}_{g, *}$, we write $\rho(\phi)^{-1}=\left(\begin{array}{ll}a & b \\ c & d\end{array}\right)$ where $a, b, c$, and $d$ are $g \times g$ integral matrices. Set

$$
A=A(\phi,[X, p, f]):=(a+\tau c)^{-1} \in G L(g, \mathbb{C})
$$

and

$$
w=w(\phi,[X, p, f]):=A^{-1} \cdot \eta(\phi \cdot[X, p, f])-\eta([X, p, f]) \in \mathbb{C}^{g} .
$$

Then Theorem 6.6 in [1] says that the vector $w$ can be decomposed into the factor from the Teichmüller space part and the factor from the mapping class group part; Namely there 
exists the uniquely determined vector $\psi(\phi) \in \frac{1}{2 g-2} \mathbb{Z}^{2 g}$ satisfying $w=(I, \tau) \psi(\phi)$. Here $I$ is the $g \times g$ identity matrix. In this way we obtain a map $\psi: \mathcal{M}_{g, *} \rightarrow \frac{1}{2 g-2} \mathbb{Z}^{2 g}$.

Moreover, Earle showed the following:

Proposition 2.1 (Earle [1]). The map $\psi$ is a twisted 1-cocycle:

$$
\psi\left(\phi_{1} \phi_{2}\right)=\rho\left(\phi_{2}\right)^{-1} \psi\left(\phi_{1}\right)+\psi\left(\phi_{2}\right), \phi_{1}, \phi_{2} \in \mathcal{M}_{g, *} .
$$

Further, when restricted to the subgroup $\pi_{1}\left(\Sigma_{g}, *\right) \subset \mathcal{M}_{g, *}, \psi$ coincides with the abelianization: $\left.\psi\right|_{\pi_{1}\left(\Sigma_{g}, *\right)}=\theta$.

In this paper we call $\psi: \mathcal{M}_{g, *} \rightarrow \frac{1}{2 g-2} \mathbb{Z}^{2 g} \cong \frac{1}{2 g-2} H$ Earle's twisted 1-cocycle.

\section{Morita's twisted 1-cocycle}

We review Morita's twisted 1-cocycle defined in [3], section 6. The abelianization $F^{a b}$ can be naturally identified with $H$ hence inherits the intersection form from $H$. For $x \in F$, We denote by $[x]$ the element of $F^{a b}=H$ represented by $x$.

Let $F(\alpha, \beta)$ be the free group generated by $\alpha$ and $\beta$. For $i=1, \ldots, g$, let $p_{i}$ be the homomorphism from $F$ to $F(\alpha, \beta)$ defined by $p_{i}\left(A_{j}\right)=p_{i}\left(B_{j}\right)=1$ if $j \neq i$, and $p_{i}\left(A_{i}\right)=\alpha$, $p_{i}\left(B_{i}\right)=\beta$. Any element $x \in F(\alpha, \beta)$ can be uniquely written as the form

$$
x=\alpha^{\varepsilon_{1}} \beta^{\delta_{1}} \cdots \alpha^{\varepsilon_{n}} \beta^{\delta_{n}},
$$

where $\varepsilon_{i}, \delta_{i} \in\{-1,0,1\}$. We first set

$$
d(x):=\sum_{k=1}^{n} \varepsilon_{k} \sum_{\ell=k}^{n} \delta_{\ell}-\sum_{k=1}^{n} \delta_{k} \sum_{\ell=k+1}^{n} \varepsilon_{\ell} .
$$

Using the same letter $d$, we next define $d: F \rightarrow \mathbb{Z}$ by

$$
d(x):=\sum_{i=1}^{n} d\left(p_{i}(x)\right) .
$$

Then the equality

$$
d\left(x x^{\prime}\right)=d(x)+d\left(x^{\prime}\right)+[x] \cdot\left[x^{\prime}\right]
$$

holds for $x, x^{\prime} \in F$. Here $[x] \cdot\left[x^{\prime}\right]$ is the intersection number of the homology classes $[x]$ and $\left[x^{\prime}\right]$. Define the map $\tilde{f}: \mathcal{N} \times F \rightarrow \mathbb{Z}$ by

$$
\tilde{f}(\phi, x)=d(\phi(x))-d(x) .
$$

Since the action of $\mathcal{N}$ on $F^{a b} \cong H$ preserves the intersection form, we see that for each $\phi \in F, \tilde{f}(\phi, \cdot)$ is a homomorphism from $F$ to $\mathbb{Z}$. Thus the map

$$
\tilde{f}: \mathcal{N} \rightarrow \operatorname{Hom}(H, \mathbb{Z}) \cong H,
$$

which will be also denoted by $\tilde{f}$, is induced. Here the isomorphism $\operatorname{Hom}(H, \mathbb{Z}) \cong H$ is Poincaré duality; $a \in H$ corresponds to the element of $\operatorname{Hom}(H, \mathbb{Z})$ given by $y \mapsto a \cdot y$. For simplicity, we write $\rho$ instead of $\rho \circ \pi: \mathcal{N} \rightarrow S p(H) \cong S p(2 g ; \mathbb{Z})$. 
Proposition 3.1 (Morita [3]). 1. The map $\tilde{f}$ is a twisted 1-cocycle:

$$
\tilde{f}\left(\phi_{1} \phi_{2}\right)=\rho\left(\phi_{2}\right)^{-1} \tilde{f}\left(\phi_{1}\right)+\tilde{f}\left(\phi_{2}\right), \phi_{1}, \phi_{2} \in \mathcal{N} .
$$

2. Let $f$ be the restriction of $\tilde{f}$ to $\mathcal{M}_{g, 1} \subset \mathcal{N}$. Then in view of the central extension (11), f factors through a twisted 1-cocycle $f: \mathcal{M}_{g, *} \rightarrow H$ (we use the same letter). Further, when restricted to the subgroup $\pi_{1}\left(\Sigma_{g}, *\right) \subset \mathcal{M}_{g, *}, f$ coincides with $(2-2 g)$ times the abelianization: $\left.f\right|_{\pi_{1}\left(\Sigma_{g}, *\right)}=(2-2 g) \theta$.

In [3], this cocycle was considered only on $\mathcal{M}_{g, 1}$. But as in the above, it can be naturally defined on $\mathcal{N}$. In this paper we call $f: \mathcal{M}_{g, *} \rightarrow H$ Morita's twisted 1-cocycle.

Regard the group $F$ as a subgroup of $\mathcal{N}$ via inner automorphism; For $x \in F$, the map $F \rightarrow F, y \mapsto x y x^{-1}$, is the corresponding element of $\mathcal{N}$.

Lemma 3.2. For $x \in F, \tilde{f}(x)=2[x]$.

Proof. Let $x, y \in F$. Using (4), we compute

$$
\begin{aligned}
\tilde{f}(x, y) & =d\left(x y x^{-1}\right)-d(y) \\
& =d(x)+d\left(y x^{-1}\right)+[x] \cdot([y]-[x])-d(y) \\
& =d(x)+d(y)+d\left(x^{-1}\right)+[y] \cdot\left[x^{-1}\right]+[x] \cdot([y]-[x])-d(y) \\
& =d(x)+d(y)-d(x)+[x] \cdot[x]+[y] \cdot\left[x^{-1}\right]+[x] \cdot([y]-[x])-d(y) \\
& =2[x] \cdot[y] .
\end{aligned}
$$

This proves the lemma.

\section{Jablow's involution}

We need the value of $\psi$ on a particular element of $\mathcal{M}_{g, *}$. Consider the endomorphism $\iota$ of $F$ defined by the following:

$$
\iota:\left\{\begin{array}{l}
A_{k} \mapsto\left(\prod_{\ell=k}^{g}\left[B_{g} \cdots B_{\ell} A_{\ell}, B_{\ell}\right] B_{\ell}\right) A_{k}^{-1}\left(\prod_{\ell=k}^{g} B_{\ell}^{-1}\right) \\
B_{k} \mapsto\left[B_{g} \cdots B_{k} A_{k}, B_{k}^{-1}\right] B_{k}^{-1}
\end{array} \quad, 1 \leq k \leq g .\right.
$$

The following can be checked by a direct computation, so we omit the proof.

Lemma 4.1. 1. The endomorphism $\iota$ is an involution of $A u t(F): \iota^{2}=\mathrm{id}$.

2. We have $\iota(\zeta)=\left(B_{g} \cdots B_{1}\right) \zeta\left(B_{g} \cdots B_{1}\right)^{-1}$. In particular, $\iota \in \mathcal{N}$.

The expression of $\iota$ is given in E. Jablow's paper [2] p.231. Thus we call it Jablow's involution. We write $\pi(\iota)=\bar{\iota} \in \mathcal{M}_{g, *}$. Note that $\rho(\bar{\iota})=-I$. Topologically, $\bar{\iota}$ is a hyperelliptic involution of $\left(\Sigma_{g}, *\right)$. 


\section{Proof of Theorem 0.1}

The following two lemmas are needed to prove Theorem 0.1;

Lemma 5.1.

$$
\tilde{f}(\iota)={ }^{t}(\underbrace{-2, \ldots,-2}_{g},-(2 g+2),-2 g, \ldots,-4) .
$$

Here we identify $H$ with $\mathbb{Z}^{2 g}$ by $H=\mathbb{Z} \cdot \theta\left(A_{1}\right) \oplus \cdots \oplus \mathbb{Z} \cdot \theta\left(B_{g}\right) \cong \mathbb{Z}^{2 g}$.

Lemma 5.2.

$$
\psi(\bar{\iota})=\frac{1}{g-1}{ }^{t}(\underbrace{1, \ldots, 1}_{g},-1,-2, \ldots,-g) .
$$

The proofs of these lemmas need fairly explicit computations and will be postponed to the next section. Using these lemmas, we can prove Theorem 0.1 .

Proof of Theorem 0.1 . Consider the twisted 1-cocycle $\psi+\frac{1}{2 g-2} f: \mathcal{M}_{g, *} \rightarrow \frac{1}{2 g-2} H$. The restriction of this cocycle to $\pi_{1}\left(\Sigma_{g}, *\right)$ is zero. Therefore, by Proposition 1.1, there exists an element $a \in \frac{1}{2 g-2} H$ such that the equation

$$
\psi+\frac{1}{2 g-2} f=\delta a
$$

holds. To determine $a$ we want to evaluate both sides on some element of $\mathcal{M}_{g, *}$. Unfortunately, $\iota$ is not an element of $\mathcal{M}_{g, 1}$ by Lemma 4.1. So $f(\bar{\iota})$ may not coincide with $\tilde{f}(\iota)$. We need to fix $\iota$ to obtain an element of $\mathcal{M}_{g, 1}$. Let $x_{B}:=\left(B_{g} \cdots B_{1}\right)^{-1} \in F \subset \mathcal{N}$. By the second statement of Lemma 4.1, $x_{B} \cdot \iota \in \mathcal{M}_{g, 1}$.

Now we compute

$$
\begin{aligned}
f\left(\pi\left(x_{B} \cdot \iota\right)\right) & =\tilde{f}\left(x_{B} \cdot \iota\right) \\
& =\rho(\iota)^{-1} \tilde{f}\left(x_{B}\right)+\tilde{f}(\iota) \\
& =-{ }^{t}(\underbrace{0, \ldots, 0}_{g}, \underbrace{-2, \ldots,-2}_{g})+{ }^{t}(\underbrace{-2, \ldots,-2}_{g},-(2 g+2),-2 g, \ldots,-4) \\
& ={ }^{t}(\underbrace{-2, \ldots,-2}_{g},-2 g,-2 g+2, \ldots,-2)
\end{aligned}
$$

by using Lemma 3.2 and Lemma 5.1 , and

$$
\begin{aligned}
\psi\left(\pi\left(x_{B} \cdot \iota\right)\right) & =\psi\left(\pi\left(x_{B}\right) \cdot \bar{\iota}\right) \\
& =\rho(\bar{\iota})^{-1} \psi\left(\pi\left(x_{B}\right)\right)+\psi(\bar{\iota}) \\
& =-{ }^{t}(\underbrace{0, \ldots, 0}_{g}, \underbrace{-1, \ldots,-1}_{g})+\frac{1}{g-1}{ }^{t}(\underbrace{1, \ldots, 1}_{g},-1,-2, \ldots,-g) \\
& =\frac{1}{g-1}{ }^{t}(\underbrace{1, \ldots, 1}_{g}, g-2, g-3, \ldots,-1)
\end{aligned}
$$

by using Lemma 5.2 and Proposition 2.1. On the otherhand, we compute

$$
\left(\psi+\frac{1}{2 g-2} f\right)\left(\pi\left(x_{B} \cdot \iota\right)\right)=\delta a\left(\pi\left(x_{B} \cdot \iota\right)\right)=\rho\left(x_{B} \cdot \iota\right)^{-1} \cdot a-a=-2 a .
$$

Combining these computations together, we obtain $a=a_{0}$. 


\section{Proofs of Lemma 5.1 and Lemma 5.2}

In this section we prove Lemma 5.1 and Lemma 5.2 ,

Proof of Lemma 5.1. Let $k \in\{1, \ldots, g\}$. By (6) , we have

$$
p_{i}\left(\iota\left(A_{k}\right)\right)= \begin{cases}1 & (i<k) \\ \beta \alpha \beta \alpha^{-1} \beta^{-1} \alpha^{-1} \beta^{-1} & (i=k) \\ \beta \alpha \beta \alpha^{-1} \beta^{-1} \beta^{-1} & (i>k)\end{cases}
$$

and

$$
p_{i}\left(\iota\left(B_{k}\right)\right)= \begin{cases}1 & (i \neq k) \\ \beta \alpha \beta^{-1} \alpha^{-1} \beta^{-1} & (i=k) .\end{cases}
$$

Direct computations according to (3) shows that

$$
d\left(\beta \alpha \beta \alpha^{-1} \beta^{-1} \alpha^{-1} \beta^{-1}\right)=4, d\left(\beta \alpha \beta \alpha^{-1} \beta^{-1} \beta^{-1}\right)=2, d\left(\beta \alpha \beta^{-1} \alpha^{-1} \beta^{-1}\right)=-2 .
$$

From these, we have $\tilde{f}\left(\iota, A_{k}\right)=4+2(g-k)$ and $\tilde{f}\left(\iota, B_{k}\right)=-2$. This proves the lemma.

We next proceed to the proof of Lemma 5.2. At first glance it seems difficult to compute the vector $w=w(\phi,[X, p, f])$ from the definition (2) because it is not easy to compute quadratic period classes in general. However, if $\phi$ is a hyperelliptic involution such as $\bar{\iota}$, all terms concerning quadratic period classes cancel and disappear as we will see in the below for the case $\phi=\bar{\iota}$.

Proof of Lemma 5.2. Let us introduce a complex structure on $\left(\Sigma_{g}, *\right)$ and denote it by $\left(X_{0}, p_{0}\right)$. Consider the point $\left[X_{0}, p_{0}, \mathrm{id}\right] \in \mathcal{T}_{g, 1}$. According to (2),

$$
w=w\left(\bar{\iota},\left[X_{0}, p_{0}, \mathrm{id}\right]\right)=-\eta\left(\left[X_{0}, p_{0}, \bar{\iota}\right]\right)-\eta\left(\left[X_{0}, p_{0}, \mathrm{id}\right]\right)
$$

since $\bar{\iota}^{-1}=\bar{\iota}$ and $A\left(\bar{\iota},\left[X_{0}, p_{0}, \mathrm{id}\right]\right)=-I$. Thus the $j$-th component of the vector $w$ is given by

$$
w_{j}=\frac{1}{g-1}\left(-\tau_{j j}+\sum_{k=1}^{g}\left(Q_{j k}\left(A_{k}\right)+Q_{j k}\left(\bar{\iota}_{*} A_{k}\right)\right)\right) .
$$

Now quadratic period classes satisfy the following property (see for instance, 2] p.222):

$$
Q_{j k}\left(T_{1} T_{2}\right)=Q_{j k}\left(T_{1}\right)+Q_{j k}\left(T_{2}\right)+\omega_{j}\left(T_{1}\right) \omega_{k}\left(T_{2}\right), T_{1}, T_{2} \in \pi_{1}\left(X_{0}, p_{0}\right),
$$

where $\omega(T)=\int_{[T]} \omega$ is the integration of the holomorphic 1-form $\omega$ along the homology class $[T]$. From this, the following formulae are easily derived;

$$
\begin{gathered}
Q_{j k}\left(T_{1} \cdots T_{m}\right)=\sum_{n=1}^{m} Q_{j k}\left(T_{n}\right)+\sum_{1 \leq n<n^{\prime} \leq m} \omega_{j}\left(T_{n}\right) \omega_{k}\left(T_{n^{\prime}}\right) \\
Q_{j k}([S, T])=\omega_{j}(S) \omega_{k}(T)-\omega_{j}(T) \omega_{k}(S) ; \\
Q_{j k}\left(T^{-1}\right)=\omega_{j}(T) \omega_{k}(T)-Q_{j k}(T),
\end{gathered}
$$


where $S, T, T_{i} \in \pi_{1}\left(X_{0}, p_{0}\right)$. We will use these formulae freely in the following computations. Set $E_{\ell}:=\left[B_{g} \cdots B_{\ell} A_{\ell}, B_{\ell}\right] B_{\ell}$. Then $\bar{\iota}_{*} A_{k}=E_{k} E_{k+1} \cdots E_{g} A_{k}^{-1} B_{k}^{-1} \cdots B_{g}^{-1}$. We compute

$$
\begin{aligned}
& Q_{j k}\left(\bar{\iota}_{*} A_{k}\right)= Q_{j k}\left(E_{k} E_{k+1} \cdots E_{g} A_{k}^{-1} B_{k}^{-1} \cdots B_{g}^{-1}\right) \\
&= \sum_{\ell=k}^{g} Q_{j k}\left(E_{\ell}\right)+Q_{j k}\left(A_{k}^{-1}\right)+\sum_{\ell=k}^{g} Q_{j k}\left(B_{\ell}^{-1}\right)+\sum_{k \leq \ell<\ell^{\prime}} \omega_{j}\left(E_{\ell}\right) \omega_{k}\left(E_{\ell^{\prime}}\right) \\
&+\sum_{\ell=k}^{g} \omega_{j}\left(E_{\ell}\right) \omega_{k}\left(A_{k}^{-1}\right)+\sum_{k \leq \ell, k \leq \ell^{\prime}} \omega_{j}\left(E_{\ell}\right) \omega_{k}\left(B_{\ell^{\prime}}^{-1}\right)+\sum_{\ell=k}^{g} \omega_{j}\left(A_{k}^{-1}\right) \omega_{k}\left(B_{\ell}^{-1}\right) \\
&+\sum_{k \leq \ell<\ell^{\prime}} \omega_{j}\left(B_{\ell}^{-1}\right) \omega_{k}\left(B_{\ell^{\prime}}^{-1}\right) ; \\
& Q_{j k}\left(E_{\ell}\right)= Q_{j k}\left(\left[B_{g} \cdots B_{\ell} A_{\ell}, B_{\ell}\right] B_{\ell}\right) \\
&=Q_{j k}\left(\left[B_{g} \cdots B_{\ell} A_{\ell}, B_{\ell}\right]\right)+Q_{j k}\left(B_{\ell}\right) \\
&=\omega_{j}\left(B_{g} \cdots B_{\ell} A_{\ell}\right) \omega_{k}\left(B_{\ell}\right)-\omega_{j}\left(B_{\ell}\right) \omega_{k}\left(B_{g} \cdots B_{\ell} A_{\ell}\right)+Q_{j k}\left(B_{\ell}\right) \\
&=\left(\sum_{m=\ell}^{g} \tau_{j m}+\delta_{j \ell}\right) \tau_{k \ell}-\tau_{j \ell}\left(\sum_{m=\ell}^{g} \tau_{k m}+\delta_{k \ell}\right)+Q_{j k}\left(B_{\ell}\right) ; \\
& Q_{j k}\left(A_{k}^{-1}\right)=\omega_{j}\left(A_{k}\right) \omega_{k}\left(A_{k}\right)-Q_{j k}\left(A_{k}\right)=\delta_{j k}-Q_{j k}\left(A_{k}\right) ; \\
& Q_{j k}\left(B_{\ell}^{-1}\right)=\omega_{j}\left(B_{\ell}\right) \omega_{k}\left(B_{\ell}\right)-Q_{j k}\left(B_{\ell}\right)=\tau_{j \ell} \tau_{k \ell}-Q_{j k}\left(B_{\ell}\right) ;
\end{aligned}
$$

$\omega_{j}\left(E_{\ell}\right)=\tau_{j \ell}$, and $\omega_{k}\left(E_{\ell}\right)=\tau_{k \ell}$. Therefore, $Q_{j k}\left(A_{k}\right)+Q_{j k}\left(\bar{\iota}_{*} A_{k}\right)$ is equal to

$$
\begin{aligned}
& \sum_{\ell=k}^{g}\left(\left(\sum_{m=\ell}^{g} \tau_{j m}+\delta_{j \ell}\right) \tau_{k \ell}-\tau_{j \ell}\left(\sum_{m=\ell}^{g} \tau_{k m}+\delta_{k \ell}\right)\right)+\delta_{j k}+\sum_{\ell=k}^{g} \tau_{j \ell} \tau_{k \ell} \\
& +\sum_{k \leq \ell<\ell^{\prime}} \tau_{j \ell} \tau_{k \ell^{\prime}}-\sum_{\ell=k}^{g} \tau_{j \ell}-\sum_{k \leq \ell, k \leq \ell^{\prime}} \tau_{j \ell} \tau_{k \ell^{\prime}}+\sum_{\ell=k}^{g} \delta_{j k} \tau_{k \ell}+\sum_{k \leq \ell<\ell^{\prime}} \tau_{j \ell} \tau_{k \ell^{\prime}} .
\end{aligned}
$$

Now no quadratic period classes appear here. We observe that the degree two terms with respect to $\tau$ (e.g. $\tau_{j m} \tau_{k \ell}$ ) appearing in (8) all cancel out. Thus (8) is equal to

$$
\sum_{k \leq \ell}\left(\delta_{j \ell} \tau_{k \ell}-\delta_{k \ell} \tau_{j \ell}\right)+\delta_{j k}-\sum_{\ell=k}^{g} \tau_{j \ell}+\sum_{\ell=k}^{g} \delta_{j k} \tau_{k \ell},
$$

and we compute $\sum_{k=1}^{g}\left(Q_{j k}\left(A_{k}\right)+Q_{j k}\left(\bar{\iota}_{*} A_{k}\right)\right)$ as

$$
\begin{aligned}
& \sum_{1 \leq k \leq \ell \leq g}\left(\delta_{j \ell} \tau_{k \ell}-\delta_{k \ell} \tau_{j \ell}\right)+\sum_{k=1}^{g} \delta_{j k}-\sum_{1 \leq k \leq \ell \leq g} \tau_{j \ell}+\sum_{1 \leq k \leq \ell \leq g} \delta_{j k} \tau_{k \ell} \\
= & \sum_{1 \leq k \leq j} \tau_{k j}-\sum_{k=1}^{g} \tau_{j k}+1-\sum_{\ell=1}^{g} \ell \tau_{j \ell}+\sum_{j \leq \ell \leq g} \tau_{j \ell} \\
= & 1+\tau_{j j}-\sum_{\ell=1}^{g} \ell \tau_{j \ell} .
\end{aligned}
$$


Here we use the symmetry condition $\tau_{k j}=\tau_{j k}$ of period matrices. Substituting (9) into (7) we obtain

$$
w_{j}=\frac{1}{g-1}\left(1-\sum_{\ell=1}^{g} \ell \tau_{j \ell}\right)
$$

and

$$
w=(I, \tau) \cdot \frac{1}{g-1}{ }^{t}(\underbrace{1, \ldots, 1}_{g},-1,-2, \ldots,-g) .
$$

Since the $2 g$ vectors of the colums of the $g \times 2 g$ matrix $(I, \tau)$ are linearly independent over the real numbers, the lemma follows.

Acknowledgments: I am grateful to Professor Nariya Kawazumi for telling me about Earle's paper, and Professor Shigeyuki Morita for useful comments about twisted 1-cocycles on the mapping class groups. I also thank them for their warm encouragements. This research is supported by JSPS Research Fellowships for Young Scientists (19.5472).

\section{References}

[1] C.J. Earle, Families of Riemann surfaces and Jacobi varieties, Ann.Math. 107 (1978) 255-286.

[2] E.R. Jablow, Quadratic vector classes on Riemann surfaces, Duke.Math. Vol.53, No.1 (1986) 221-232.

[3] S. Morita, Families of Jacobian manifolds and characteristic classes of surface bundles I, Ann.Inst.Fourier 39 (1989) 777-810.

[4] S. Morita, Families of Jacobian manifolds and characteristic classes of surface bundles II, Math.Proc.Camb.Phil.Soc 105 (1989) 79-101.

[5] S. Morita, Casson's invariant for homology 3-spheres and characteristic classes of surface bundles I. Topology 28 (1989) 305-323.

[6] S. Morita, Casson invariant, signature defect of framed manifolds and the secondary characteristic classes of surface bundles, J.Diff.Geom 47 (1997) 560-599.

[7] R. Trapp, A linear representation of the mapping class group $\mathcal{M}$ and the theory of winding numbers, Topology and its Applications 43 (1992) 47-64.

YUSUKE KUNO

Graduate School of Mathematical Sciences,

The UnIVERSITY OF TOKYO,

3-8-1 Komaba Meguro-Ku Tokyo 153-0041, JAPAN

E-mail address:kunotti@ms.u-tokyo.ac.jp 